\title{
STUDIES ON THE RELATIONSHIP OF PROTEIN BINDING AND GEL-AFFINITY OF ANTIBIOTICS INCLUDING PENICILLINS
}

\author{
Takeo Murakawa, Yoshimi Wakat and Minoru Nishida \\ Research Laboratories, Fujisawa Pharmaceutial Co., Ltd., \\ Osaka, Japan
}

(Received for publication August 4, 1970)

\begin{abstract}
This paper concerns the relationship between the extent of serum-protein binding of antibiotics and the corresponding effluent volume (Ve), and the relationship between the protein-binding extent and the gel-affinity. In the study of the former relationship, the $V e$ values of penicillins and other antibiotics were estimated by gel filtration. Compounds showing an increase in serum binding also showed an increase in the Ve value. Accordingly, the extent of binding to serum proteins could be predicted without using serum. In studying the latter, the batchwise method was used, by which, a similar relationship between the extent of protein binding and the corresponding gelaffinity was elucidated.
\end{abstract}

The binding of penicillins and other antibiotics to serum proteins has been extensively studied ever since this phenomenon was discovered. ${ }^{1,2,3)}$ In recent years a large number of reports have been offered, each concerning principles and concept fundamental to the interpretation of this phenomenon. However, there is only a paucity of information on the mode of binding and its physiological and pharmacological significance. The gel filtration technique has recently been applied to the study of the interaction between proteins and low-molecular weight compounds. ${ }^{4,5,6,7,8)}$ GELOTTE et al. showed that most aromatic compounds were reversibly adsorbed on Sephadex gel. ${ }^{9,10,11)}$ It is also known that, not only the hydroxyl group contained in gel materials, but polymers such as polyacrylamide have also affinity for aromatic and pseudoaromatic compounds.

This paper concerns the behavior of various penicillins and other antibiotics on gel-filtration with a view to clarifying the mode of their protein-binding.

\section{Materials}

Human serum albumin (96\% pure, chromatographically isolated) was obtained from Mann Research Laboratories, U.S.A.

The following antibiotics were used: Benzyl penicillin, ampicillin and cloxacillin from Beecham Laboratories; oxacillin and dicloxacillin from Bristol Laboratories; tetracycline, oxytetracycline and methacycline from Pfizer Laboratories; cephalexin and cephalothin from Eli Lilly Laboratories; cephaloridine from Glaxo Laboratories; cefazolin from Fujisawa Pharmaceutical Co., Ltd.

Sephadex G-10, G-25 and G-50, and Blue Dextran 2,000 were obtained from Pharmacia Fine Chemicals Ltd., and Bio-gels P-2, P-4 and P-6 from Bio-Rad Laboratories. 


\section{Methods}

1. Determination of Ve value on gel filtration

Sephadex gels or Bio-gels of various pore sizes were allowed to swell in Sörensen's $1 / 15 \mathrm{M}$ phosphate buffer, $\mathrm{pH} 7.0$, at room temperature overnight and packed into a column (internal diameter $2.5 \mathrm{~cm}$, Pharmacia Fine Chemicals Ltd.) to give a gel bed-depth of 15 $25 \mathrm{~cm}$. The packed gels were equilibrated for about 3 hours with the buffer supplied at the rate of $100 \sim 150 \mathrm{ml}$ per hour by a peristaltic pump.

Gel filtration of antibiotics was performed under the following conditions: Eluent, SöRENSEN's $1 / 15 \mathrm{M}$ phosphate buffer; sample, $1 \mathrm{ml}(1 \sim 3 \mathrm{mg} / \mathrm{ml})$; flow rate, $100 \sim 150 \mathrm{ml} /$ hour.

The effluent solution was continuously monitored through a quartz flow cell in the Ohtake UV Auto spectrophotometer at $255 \mathrm{~m} \mu$. The Ve value represents the volume of the effuent required to obtain the maximum antibiotic concentration through the gel filtration.

2. Determination of protein binding

The centrifugal ultrafiltration technique was used to determine the extent of binding. Solutions of antibiotics were prepared with the buffer so as to give various concentrations. A $0.5-\mathrm{ml}$ aliquot of an antibiotic solution was added to $4.5 \mathrm{ml}$ of human albumin solution ( $1 \mathrm{~g}$ of human albumin was dissolved in $90 \mathrm{ml}$ of the buffer). The mixture obtained was incubated at $37^{\circ} \mathrm{C}$ for 1 hour. A reference experiment was performed by use of the buffer in place of the albumin solution. Bags for centrifugal ultrafiltration were prepared from Visking cellulose tubing (Visking Company, 8/32 in size). Each of the bags containing $2 \mathrm{ml}$ of the mixture or reference mixture was hung in a $15-\mathrm{ml}$ polypropylene tube. Tubes containing the bag were centrifuged at $1,000 \times \mathrm{g}$ for about 40 minutes. The antibiotic content of the resultant ultrafiltrate was determined by the paper disc method using Bacillus subtilis ATCC 6633 as the test organism. The extent of binding of an antibiotic to albumin was calculated by the equation provided below:

$$
\text { per cent bound }=\frac{R-S}{S} \times 100
$$

where $S$ is the antibiotic content in the ultrafiltrate of samples and $R$ is the content in the ultrafiltrate of the reference.

\section{Results}

1. Relationship between the Ve value in Sephadex gel-filtration and protein-binding

Antibiotics used in the present experiment were selected from the considerable number available and differed in their extent of protein binding. The relation between the Ve value using Sephadex G-25 and the extent of protein binding is shown in Fig. 1. It will be seen that a close relationship was obtained between the two variables: the Ve value of ampicillin (93) was accompanied by a low proteinbinding property, while that of dicloxacillin (as high as 168) was accompanied by a considerably higher protein-binding. Furthermore, studies revealed that, at human albumin concentrations of both $1 \%$ and $4 \%$, with all the penicillins tested an increase in protein binding was accompanied by an increase in the Ve values. Despite the similarity of their molecular weights, the Ve values of these penicillins differed significantly from one other. These results suggested that penicillins with higher Ve values might penetrate the gel net-work more efficiently and/or be adsorbed in the gel matrix. 
Fig. 1. Relationship between the Ve value in Sephadex gel filtration and protein binding.

1) Penicillins

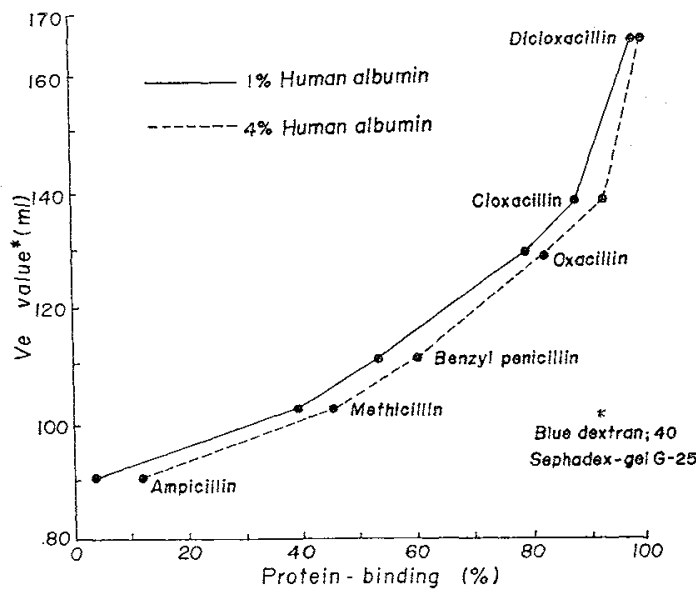

The above relationship obtained from penicillins proved so interesting that the authors conducted similar experiments on cephalosporins and tetracyclines under the same conditions. The results are shown in Fig. 2. The relationship between the $\mathrm{Ve}$ value and the extent of protein binding was similar to that with penicillins.

2. Influence of pore size on the relationship between the $\mathrm{Ve}$ value and protein-binding extent

It has been recognized that molecular sieving is an important factor in characterizing gel filtration and is regulated by the pore sizes of gels used. In this experiment, the Ve values of penicillins were determined on Sephadex
Fig. 2. Relationship between the Ve value in Sephadex gel filtration and protein binding.

2) Tetracyclines and cephalosporins

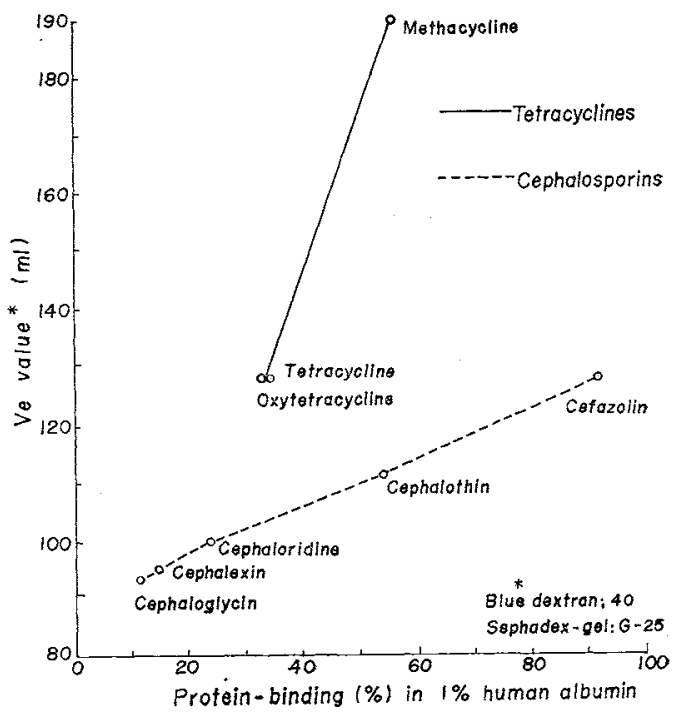

Fig. 3. Influence of pore size on relationship between the $\mathrm{Ve}$ value and protein-binding extent.

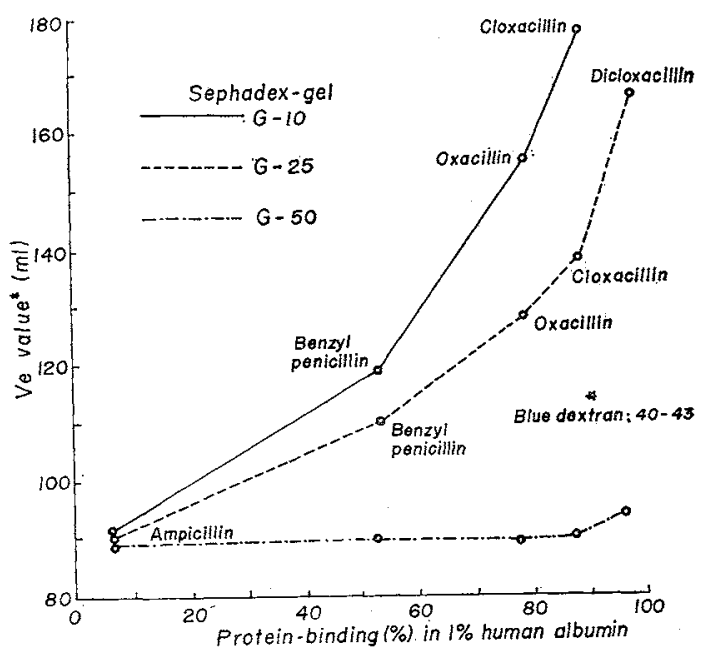
gels, not only on G-25 but also on the $G-10$ and $G-50$, the pore sizes of which differed from those of G-25. Each Ve value of the penicillins was plotted against respective value for protein-binding. As shown in Fig. 3, the Ve values of the penicillins varied according to the kind of Sephadex gels: G-10, having the fractional range of $\mathrm{M.W}$. up to $700 ; \mathrm{G}-25,100 \sim 5,000$; and $\mathrm{G}-50,500 \sim 10,000$.

In the case of $\mathrm{G}-10$, the extent of protein binding of each penicillin was well correlated with the corresponding Ve values, being better proportioned than when the $\mathrm{G}-25$ was used. In the case of $G-50$, the $V e$ values of all the penicillins tested were approximately of the same level (90), and were accordingly independent of the 
extent of protein binding.

This fact indicates that the Ve values of penicillins are dependent on the molecular sieving effect, and that this relationship applies when the pore size of the gel net-work is small.

3. Influence of $\mathrm{NaCl}$ concentration on the relationship between the Ve value and extent of protein-binding

In discussing the interaction between the gel and solute, aside from the molecular sieving effect, aromatic affinity should be taken into consideration as another factor. It is well known that the aromatic affinity is affected by the constituents of the eluent, aromatic affinity of the gel used is generally reduced. On the other hand, when eluents containing $\mathrm{NaCl}$ are used no definite tendency is seen. In general, however, the $\mathrm{Ve}$ value increases under such conditions as the aromatic affinity is intensified in the course of gel filtration. Considering the above concept, the effect of $\mathrm{NaCl}$ concentration on the Ve value of penicillins was investigated.

As is shown in Fig. 4, when $\mathrm{NaCl}$ solution was used in place of the phosphate buffer as the eluent, the $V e$ values of penicillins increased at a high concentration (3M), more markedly than at a lower concentration ( $1 \mathrm{M})$. This phenomenon reminded us of the results with phosphate buffer (Fig. 1), in which the Ve values obtained from gel filtrations with G-25 and G-10 systems closely resembled the values obtained with $1 \mathrm{~m}$ and $3 \mathrm{~m}$ NaCl. Meanwhile,
Fig. 4. Influence of $\mathrm{NaCl}$ concentration on the relationship between the Ve value and protein-binding extent.

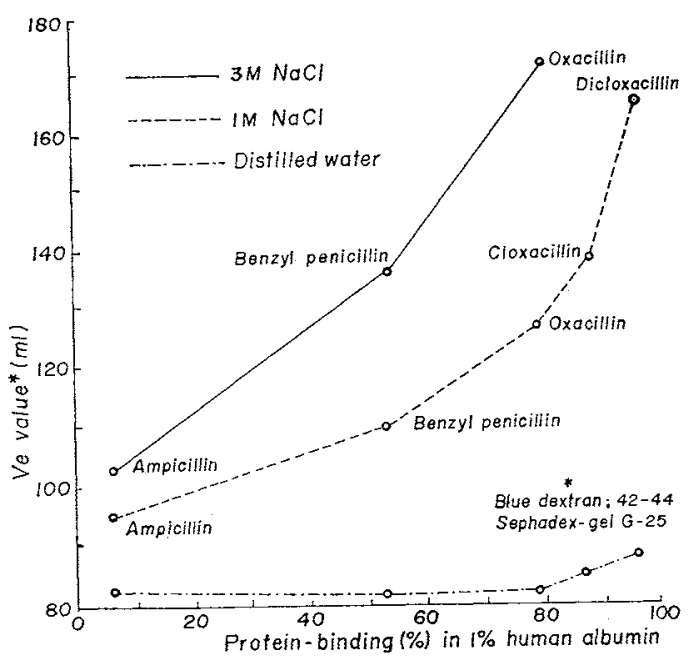

Fig. 5. Relationship between the Ve values and extent of protein binding when other types of gel were used.

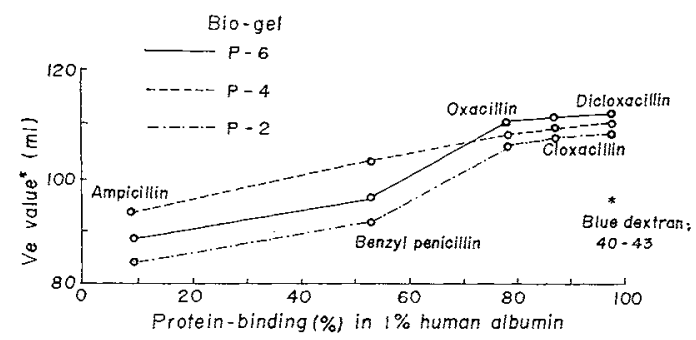

Fig. 6. Behavior of penicillins on gel by batchwise method, and protein binding.

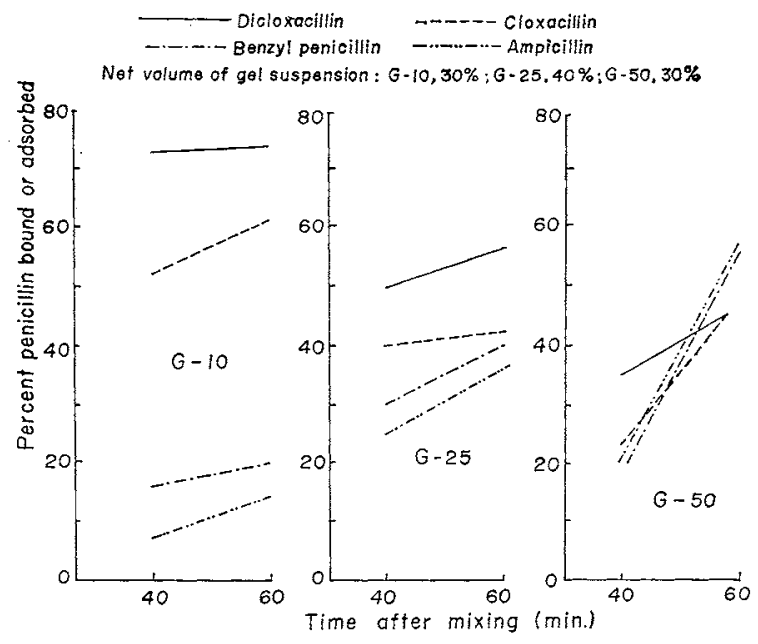


when distilled water was used as the eluent, little difference was found among the Ve values of the penicillins tested.

These findings indicate that an appropriate salt concentration is required to obtain the Ve values best correlated with the true extent of protein-binding of penicillins.

4. Relationship between Ve values and extent of protein-binding when other types of gel were used

Sephadex, chemically a cross-linked dextran, possesses a favorable property for gel filtration. There are many kinds of Sephadex available which differ in the extent of swelling. Other synthetic gels are also available for gel filtration. For example, Bio-gel, a co-polymerizate of acrylamide and methylenebisacrylamide, is useful because it covers a similar molecular size range as does Sephadex.

As in the experiments with Sephadex, the Ve values of penicillins were determined similarly with the use of three Bio-gel systems, each intended for fractionation of specified M.W. range, viz. P-2 for $200 \sim 2,000, \mathrm{P}-4$ for $500 \sim 4,000$, and $\mathrm{P}-6$ for $1,000 \sim$ 5,000 .

As shown in Fig. 5, the Ve values on the Bio-gel did not correlate so well with the extent of protein binding of penicillins as was the case with Sephadex G-25, and the results closely resembled those obtained with Sephadex G-50.

These findings indicate that the correlation between the Ve value and the extent of protein-binding is dependent not only on the pore size of the gel but also on the chemical properties of the gel used.

5. Behavior of penicillins on gel by batchwise method, in relation to protein-binding

In order to estimate the direct interaction of penicillins with Sephadex gel materials, the amount of penicillins bound (or adsorbed) was determined on the batchwise method.

As described in (2), gel filtration by the use of G-50 gave no distinct relationship between the Ve value and the extent of protein-binding. As shown in Fig. 6, four penicillins differing in the extent of protein-binding did show significant differences in their affinity for Sephadex gel G-50. On the other hand, with Sephadex gel G-10, in which gel filtration correlated closely with protein-binding, it was found that the affinity for the gel also varied with the extent of protein-binding: highly proteinbound penicillins used were cloxacillin and dicloxacillin, and penicillins with a low level of binding were ampicillin and benzylpenicillin. These results were comparable to those obtained from gel-filtration when the same gel was used.

Furthermore, a similar batchwise experiment was carried out with Sephadex gel G-25 in place of G-10. In this case, the relative affinity of each penicillin for the gel were in exactly the same order as with G-10, but the differences in the affinity of the penicillins for the gel were less marked than when $G-10$ was used.

\section{Discussion}

In the present investigation, an interesting relationship was found between the protein-binding of antibiotics and Ve values on gel filtration by Sephadex G-10 and G-25. 
With each of the antibiotics used, the Ve value correlated well with the extent of protein binding, and the $\mathrm{Ve}$ value increase with increase in protein binding.

As is well known, the molecular sieving effect and aromatic affinity are closely related in the mechanism of gel-filtration. In view of this point, despite the similarity of molecular sizes and weights of the antibiotics tested, differences in Ve values of those antibiotics indicate that the molecular sieving effect is excluded from the relationship between the $\mathrm{Ve}$ value and protein-binding. This concept was supported by the fact that no such relationship was found in experiments using either distilled water as an eluent or Bio-gel as a gel material. Accordingly, it may be justifiably concluded that, as far as these antibiotics tested are concerned, the aromatic affinity may be the main factor in this interesting relationship, as well as the pore size of gels.

Helmut et al. ${ }^{11)}$ who studied the nature of aromatic affinity by the use of three kinds of Sephadex gels, concluded that the sorption of phenol increased in the order of G-25, $\mathrm{G}-15$, and $\mathrm{G}-10$, and that the sorption was probably due to ether-linkage in the gel matrix. The authors obtained similar results with penicillins.

Whilst the mode of protein-binding of antibiotics remains obscure, the particular chemical structures and configurations required for the binding have been elucidated to a great extent.

Our studies on the relationship between the $\mathrm{Ve}$ value and the extent of protein binding have revealed that both the albumin-binding and the gel-interaction of antibiotics are based on similar mechanisms. Sephadex gels are copolymerizate of epichlorohydrin and polysaccharide dextran through an ethereal linkage, and by no means related to the albumin in structure. The conclusion that aromatic affinity is the principle factor in the similarity between the gels and albumin with respect to their interaction with antibiotics, satisfactorily accounts for the fact that Sephadex gel material and albumin have a common chemical property in binding antibiotics.

The following speculation might be proposed to interpret the results:

(1) The structure of gels, forming a high cross-linkage with ether-groupings, provides the gels with a specific hydrophobic character which is responsible for the antibiotic affinity.

(2) The extent of the aromatic affinity is dependent upon the side structure at the $\mathrm{C}_{6}$-(penicillins) or $\mathrm{C}_{7}$-positions (cephalosporins).

(3) The specificity of antibiotics in the protein-binding is expressed in terms of their interaction with the Sephadex gel.

\section{Acknowledgement}

The authors wish to thank Dr. H. NAKano and Dr. S. Kumada of Fujisawa Research Laboratories for guidance and encouragement.

\section{Bibliography}

1) Rolinson, G. N. \& R. Sutherland: The binding of antibiotics to serum proteins. Brit. J. Pharm. 25..638 650, 1965

2) Murakawa, T.; Y. Wakai, Y. Doi \& M. Nishida: Studies on binding of penicillins to serum protein. Jap. J. Antibiotics $22: 387 \sim 393,1969$

3) Meyer, M. C. \& D. E. Guttman: The binding of drugs by plasma proteins. (Review Article) J. Pharmaceut. Sci. $57: 895 \sim 918,1968$

4) Hummel, J. P. \& W. J. Dreyer: Measurement of protein-binding phenomena by gel filtration. Biochim. Biophys. Acta $63: 530 \sim 532,1962$

5) HARDY, T.L. \& K.R.L. MANSFORD: Gel filtration as a method of studying drug-protein binding. Biochem. J. $83: 34 \sim 35,1962$

6) Acred, P.; D. M. Brown, T. L. Hardy \& K.R.L. Mansford: A new approach to studying the protein binding properties of penicillins. Nature $199: 758 \sim 759,1963$ 
7) Ashworth, R.W. \& D. D. Heard: The evaluation of a molecular sieve technique to determine the interaction between a preservative and surfactant. J. Pharm. Pharmacol. 18 (Suppl.): 98s $102 \mathrm{~s}, 1966$

8) Cooper, P.F. \& G. C. Wood: Protein-binding of small molecules, new gel filtration method. J. Pharm. Pharmacol. 20 (Suppl.): 150s 156s, 1968

9) Porath, J.: Gel filtration of proteins, peptides and amino acids. Biochem. Biophys. Acta 39 : 193,1960

10) Schwarz, A. N.; A.W.G. YFe \& B. A. Z Z ABIN: Separation of nucleotides, nucleosides and bases on a new gel filtration material. J. Chromatogr. $20: 154,1965$

11) Determann, H. \& I. Walter: Source of aromatic affinity to Sephadex Dextran gel. Nature $219: 604 \sim 605,1968$ 